\title{
Croissance hivernale et printanière de prairies permanentes pâturées en montagne. I. - Ecophysiologie du dactyle
}

\author{
Michel DURU \\ avec la collaboration technique de Louis SOS $\left({ }^{*}\right)$ \& Roger VIARD $(*)$
}

I.N.R.A., Station d'Agronomie (*) et Unité de Recherches sur les Systèmes Agraires et le Développement, Centre de Recherches de Toulouse, B.P. 27, F 31326 Castanet-Tolosan

Mots clés additionnels : Dactylis glomerata, morphogenèse, température, tallage, élongation et apparition des feuilles, nutrition minérale, défoliation.

In winter and spring, the morphogenesis of cocksfoot in permanent pastures was studied at different soil fertility levels to answer the two following questions. The first question was whether PK nutrition or botanical composition could explain the major differences in growth rate previously observed between plots. In the case of cocksfoot (chosen as an example), we showed a great effect of PK nutrition. The second question concerned the interaction between climate, plant nutrition and defoliation, particularly in certain poorly understood situations : low temperature, snow cover, and low soil fertility level. Mineral deficiencies $(\mathrm{P}, \mathrm{K}$ and $\mathrm{N})$ had large effects after the beginning of February, when grasses are at the reproductive stage. Defoliation reduced most growth components, principally after the beginning of February. Despite the very different and non-linear effects of temperature on lamina extension, leaf appearance rate and tillering, we observed that, for a given plant nutrition level, tuft growth in relation to temperature was approximatiwely linear. We suppose that regulatory processes operate within the tuft.

Additional key words : Dactylis glomerata, morphogenesis, temperature, lamina extension, leaf appearance rate, tillering, mineral plant nutrition, defoliation.

\section{INTRODUCTION}

L'importance du pâturage dans l'alimentation hivernale (début novembre à fin avril) des troupeaux ovins pyrénéens (DURU et al., 1979 ; GIBON, 1981) nous a conduit à caractériser les disponibilités au pâturage à savoir les réserves sur pied et la croissance proprement dite (DURU \& GIBON, 1981). Nous avons montré que la croissance est continue pendant l'hiver, même lorsque les conditions climatiques sont défavorables (DURU, 1983). La croissance est alors très dépendante de la température (dont l'effet est diffé- 
rent entre le début et la fin de l'hiver) et de la nutrition minérale principalement en fin d'hiver et au printemps (DuRU, 1985).

Nous avons choisi d'étudier l'écophysiologie du dactyle considéré comme plante témoin d'un couvert végétal plurispécifique pour tester 2 hypothèses. En premier lieu, les effets de la nutrition minérale ont été étudiés à partir d'observations de la croissance sur des parcelles de fertilité phosphopotassique et de flore différentes de telle sorte que les écarts de croissance observés peuvent provenir de l'un ou de l'autre de ces facteurs.

D'autre part, il importe de vérifier dans quelle mesure le fait de considérer des relations linéaires entre croissance et température pour un niveau de nutrition minérale donné (DURU, 1985) ne simplifie pas trop la réalité sachant que les effets de la température et de la nutrition minérale sont différents sur l'élongation des limbes, le rythme d'apparition des feuilles et le tallage. Cette vérification est particulièrement nécessaire pour les cas de figures mal connus à savoir très faibles températures, sols très carencés en éléments minéraux et prairies pâturées. Ce sera l'objet de cette première partie.

L'étude de la croissance, au niveau global du couvert végétal, en fonction du climat et des conditions de nutrition minérale, sera reprise dans une deuxième partie et discutée en fonction des conclusions concernant l'écophysiologie du dactyle. L'objectif est d'estimer les disponibilités pour le pâturage et leur valeur alimentaire à partir de l'étude de la composition chimique.

\section{MATÉRIEL ET MÉTHODES}

\section{A. Site}

Les observations et contrôles ont été réalisés sur 2 prairies permanentes situées à $1250 \mathrm{~m}$ dans la vallée du Larboust (Pyrénées). Il s'agit de deux parcelles qui sont utilisées en pâture de début novembre à fin avril et fauchées 2 fois durant l'été. Elles représentent 2 niveaux de fertilité chimique résultant de leur histoire culturale (BALENT \& DURU, 1984). Dans la suite du texte nous caractériserons ces 2 parcelles par les initiales suivantes : B bonne, $\mathrm{M}$ moyenne. La composition floristique a été mesurée par la méthode des points quadrats (DAGET \& POISSONNET, 1971) et ces résultats ainsi que les analyses de terre sont indiqués dans le tableau 1. Le dactyle est présent à l'état de touffe. Ces parcelles reçoivent une fumure organique, mais aucune fertilisation phosphopotassique minérale.

\section{B. Climat}

Nous avons enregistré la température quotidienne à $10 \mathrm{~cm}$ dans le sol et $150 \mathrm{~cm}$ au-dessus du sol. L'enneigement au sol a été relevé. Pour les 4 hivers étudiés $(81 / 82,82 / 83,83 / 84,84 / 85)$ ces données climatiques sont représentées figure 1 . On note un enneigement moyen de 48 à 66 jours pour les 4 années, les extrêmes observés sur 20 ans étant de 15 à 110 jours. Les températures observées à $-10 \mathrm{~cm}$ ont été variables; on note des écarts de $2{ }^{\circ} \mathrm{C}$ entre années au début et à la fin de l'hiver.

TABLEAU

Analyse de terre et composition floristique des trois parcelles étudiées.

Soil analysis and botanical composition of the three plots studied.

\begin{tabular}{lllll}
\hline Parcelles & \multicolumn{2}{c}{ B } & \multicolumn{2}{c}{ M } \\
\hline $\begin{array}{l}\text { Analyse de terre } \\
\text { (horizon en cm) }\end{array}$ & $0-15$ & $15-25$ & $0-15$ & $15-25$ \\
\hline Azote Kjeldahl \%oo & & & & \\
Acide phosphorique \%o & 5,13 & 3,20 & 5,20 & 5,00 \\
Potasse échangeable \%o & 0,108 & 0,051 & 0,045 & 0,030 \\
Matière organique \% & 0,43 & 0,28 & 0,15 & 0,09 \\
C/N & 7,0 & 3,6 & 5,9 & 5,4 \\
& 7,9 & 6,5 & 6,6 & 6,2 \\
\hline
\end{tabular}

Composition floristique en $\%$

- bonnes graminées

(dont dactyle)

- graminées médiocres

- legumineuses

- diverses fourragères

- diverses non fourragères

\section{$33(15)$}

$30(29)$

\section{Observations réalisées}

Les contrôles ont été réalisés de début novembre à fin avril toutes les 3 semaines ou plus en cas d'enneigement au sol. Les dates d'observations sont indiquées sur la figure 1a. Il a été réalisé en moyenne 6 observations par hiver.

Trois traitements azotés ont été appliqués selon la date et le nombre d'apports d'azote (tabl. 2). Sur la parcelle $\mathrm{B}$, un traitement complémentaire appelé $\mathrm{P}$, simulant la pâture correspond à une défoliation complète des plantes observées.

\section{Croissance et sénescence des limbes}

Des séries de 20 feuilles de dactyle ont été identifiées par baguage au stade "feuille pliée » lorsque le limbe dépassait de la gaine d'environ $12 \mathrm{~mm}$. A chaque observation, la longueur et l'état des limbes ont été notés et une nouvelle série d'observations a été mise en route.

\section{Rythme d'apparition des feuilles, variations $d u$ nombre de talles}

En début de campagne, nous avons repéré 20 touffes de dactyle par traitement soit environ 100 à 170 talles selon les cas. A chaque date d'observation, nous épointons les feuilles présentes de telle sorte qu'à la date suivante nous puissions comptabiliser les feuilles nouvellement apparues. Pour le traitement P, la totalité des feuilles est prélevée à chaque observation (la feuille est sectionnée à environ $10 \mathrm{~mm}$ de la base).

A chaque date, le nombre de talles vivantes observées est la résultante des talles apparues et disparues. Le taux réel de tallage est donc sous-estimé du moins à certaines périodes.

\section{Croissance par touffe}

A chaque observation et pour chaque touffe, la longueur de chaque feuille nouvellement apparue a été estimée visuellement. Cette estimation peut être consi- 
$1 \mathrm{a}$

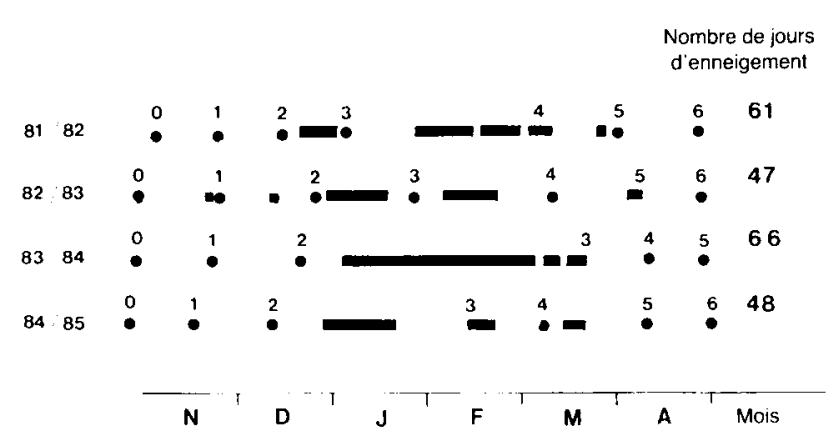

Dates d'observation de la figure $1 a$.

\begin{tabular}{|c|c|c|c|c|c|c|c|c|}
\hline & \multicolumn{8}{|c|}{$\mathrm{N}^{0}$ d'observation } \\
\hline & & 0 & 1 & 2 & 3 & 4 & 5 & 6 \\
\hline \multirow{4}{*}{ Années } & $(81 / 82$ & $3 / 11$ & $24 / 11$ & $14 / 12$ & $5 / 1$ & $4 / 3$ & $30 / 3$ & $26 / 4$ \\
\hline & $82 / 83$ & $28 / 10$ & $23 / 11$ & $17 / 12$ & $27 / 1$ & $10 / 3$ & $6 / 4$ & $27 / 4$ \\
\hline & $83 / 84$ & $26 / 10$ & $21 / 11$ & $19 / 12$ & $19 / 3$ & $10 / 4$ & $26 / 4$ & \\
\hline & $84 / 85$ & $23 / 10$ & $14 / 11$ & $10 / 12$ & $13 / 2$ & $7 / 3$ & $9 / 4$ & $29 / 4$ \\
\hline
\end{tabular}

$1 b$

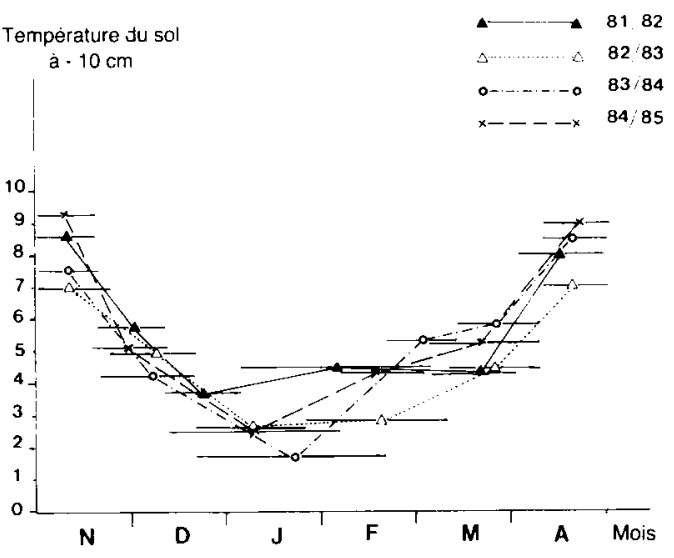

Figure 1

Caractéristiques du climat des années étudiées.

Enneigement au sol et numérotation chronologique des dates d'observation $(0,1,2, \ldots, 6)$ (fig. Ia).

Température à - $10 \mathrm{~cm}$ (fig. $1 \mathrm{~b})$.

Characteristics of climate for years studied.

Snow cover and chronological numbering of period of observa-

tion $(0,1,2, \ldots, 6)$ (fig. la).

Soil temperature $(-10 \mathrm{~cm})(\mathrm{fig} . \mathrm{lb})$

\section{TABLEAU 2}

Fertilisation et modalités de défoliation selon les parcelles et les années. $B, M$ : parcelles non défoliées; BP : parcelle où l'ensemble des feuilles des talles observées sont prélevées à chaque contrôle; No : pas de fertilisation azotée; $N 0+60: 60 \mathrm{~kg}$ de $\mathrm{N}$ début mars; N60 + 60 : $60 \mathrm{~kg}$ de $N$ à l'automne et début mars.

Fertilization and defoliation management according to plot and year. $B, M:$ plots without defoliation; $B P:$ plot $B$ with complete defoliation of every tiller at every observation; NO: no nitrogen fertilization; NO $+60: 60 \mathrm{~kg}$ of nitrogen at the beginning of March;N60+60:60 kg of nitrogen in the autumn and at the beginning of March.

\begin{tabular}{|c|c|c|c|c|c|c|c|}
\hline Parcelles & & M & & & B & & $\mathrm{BP}$ \\
\hline Azote & No & $\mathrm{N} 0+60$ & $N 60+60$ & No & $\mathrm{N} 0+60$ & $\mathrm{~N} 60+60$ & $N 60+60$ \\
\hline $81 / 82$ & $x$ & & & & & & \\
\hline $82 / 83$ & $\mathrm{x}$ & $x$ & & $\mathrm{x}$ & $\mathrm{x}$ & & \\
\hline $83 / 84$ & & & & $\mathrm{x}$ & $\mathrm{x}$ & $\mathrm{x}$ & $\mathrm{x}$ \\
\hline $84 / 85$ & & & $\mathrm{x}$ & $\mathrm{x}$ & $\mathrm{x}$ & $\mathrm{x}$ & $\mathrm{x}$ \\
\hline
\end{tabular}

dérée comme fiable car elle a toujours été réalisée après étalonnage et par le même observateur. L'intérêt de cette mesure est d'intégrer les variations d'élongation des feuilles, de leur rythme d'apparition et du tallage. Cette donnée constitue donc un bon indicateur de la croissance. Pour comparer les traitements et les années, nous avons indicé à 100 le nombre de talles au premier contrôle.

\section{RÉSULTATS}

\section{A. Croissance des limbes}

Nous avons calculé l'accroissement journalier moyen des feuilles restées vertes entre $t_{0}$ et $t_{1}$ ( $t_{0}$ étant la date à laquelle les feuilles sont baguées et $t_{1}$ la date d'observation suivante). L'analyse à une date donnée de la dispersion de ces accroissements au sein des 20 répétitions montre une répartition unimodale pour les dates d'observation de début d'hiver mais bimodale en fin d'hiver et tout particulièrement pour les placettes ayant reçu une fertilisation azotée. Dans ce cas, 10 à $25 \%$ des feuilles ont un accroissement très inférieur ( $1 / 3$ environ).

Cette dispersion peut dépendre d'un échantillonnage représentant mal la structure du peuplement puisque nous savons par ailleurs que l'élongation des feuilles peut dépendre du type de talles (BRERETON et al., 1985) ou du rang d'une feuille sur une talle (RoBSON, 1974). Cependant, compte tenu de l'importance des dispersions et de la période où nous les avons observées, on peut penser qu'il s'agit plutôt d'un effet de la vernalisation. En effet dans la mesure où ce processus s'étend sur une longue période, toutes les talles présentes dans la $2^{\mathrm{e}}$ partie de l'hiver n'ont pas été vernalisées (GIllet, 1967 ; Brereton et al., 1985). Pour les calculs suivants, nous n'avons retenu que les répétitions correspondant au deuxième mode. 
De nombreux travaux (PEACOOK, 1975 ; Behague, 1978 ; PARSON \& ROBSON, 1980) ont montré pour les graminées, l'influence différenciée de la température en automne et au printemps ; ce changement découlant du passage de la graminée du stade végétatif au stade reproducteur. Dans le cas présent, l'observation graphique de la répartition des coordonnées des observations (élongation des limbes par jour, température du sol) suggère que dans nos conditions ce changement se situe vraisemblablement début février. Nous avons calculé pour chacune de ces périodes (a : "automne" avant mi-février et $\mathrm{p}$ : «printemps" après cette date) des relations du type $\mathrm{e}=\alpha \mathrm{e}^{\beta \theta}$ (où $\mathrm{e}$ est l'élongation par limbe et par jour $\alpha$ et $\beta$ des coefficients et $\theta$ la température du sol à $-10 \mathrm{~cm}$ ). Les coefficients de la régression sont indiqués tableau 3 et les courbes sont représentées figures $2 \mathrm{a}$ et $2 \mathrm{~b}$ (sauf pour la parcelle $\mathrm{B}$ défoliée). Pour la parcelle $\mathrm{B}$, on constate que les coefficients $\beta$ sont toujours plus élevés au début du printemps et les coefficients $\alpha$ plus élevés lorsqu'il y a apport d'azote. Ceci rejoint les conclusions de LEMAIRE (1985) qui indique que le coefficient $\beta$ traduirait un effet saison et $\alpha$ dépendrait du niveau de nutrition azotée et du type génétique. En poursuivant ce raisonnement, nous devrions avoir des coefficients $\alpha$ inférieurs pour la parcelle $M$ ce que nous n'observons pas. Nous pouvons penser que la valeur des coefficients $\alpha$ et $\beta$ est particulièrement peu fiable dans ce cas précis compte tenu des faibles élongations mesurées $(0,1$ à $3,1 \mathrm{~mm} / \mathrm{j})$ et donc des incertitudes qui en découlent (les 2 coefficients étant liés dans une régression linéaire). Les différences maximales entre traitement (azote apporté, fertilité du sol) s'observent à la période où le potentiel de croissance des feuilles est le plus important (après mi-février).

\section{B. Rythme d'apparition des feuilles}

Le rythme d'apparition des feuilles a été calculé par talle vivante. Nous observons, de même que pour l'élongation des limbes, des différences importantes avant et après mi-février aussi bien pour la parcelle $B$ que la parcelle $M$. Pour les données de cette dernière période, nous notons une tendance asymptotique. A faible température $\left(5^{\circ} \mathrm{C}\right)$, nous observons des valeurs élevées du rythme d'apparition des feuilles et ce pour des relevés indépendants (avec et sans azote). Cette dynamique peut être un artéfact lié à la faible gamme de température explorée.

Les coefficients de régression (tabl. 3) montrent que l'effet de l'azote apparaît faible aux basses températures mais non négligeable aux températures supérieures. La fertilité du sol semble avoir un effet net surtout après mi-février. Ce constat rejoint les observations de Gillet (1969) qui montre un effet de la nutrition sur la vitesse d'émission des feuilles.

Pour les placettes défoliées (parcelle B), on a observé une réduction importante du rythme d'apparition des feuilles (environ $30 \%$ ) en accord avec les résultats de DAviEs (1974) mais à une autre période de l'année.

\section{Sénescence}

D'une manière générale, la durée de vie d'une feuille dépend de la température pendant sa croissance (GILlet, 1980 ; Vine, 1983). Compte tenu de notre méthode d'observation, nous avons retenu le critère suivant pour apprécier la sénescence :

$\mathrm{S}=\frac{\text { longueur cumulée de feuilles jaunes et sèches }}{\text { longueur totale des feuilles observées }}$

Cette donnée n'est disponible que pour les hivers 81/82 (parcelle M), 82/83 (M et B), 83/84 (B). Nous avons trouvé comme LEMAIRE (1985) une meilleure corrélation avec la température aérienne qu'avec la température du sol. Les données de la figure 3 montrent une très grande dispersion du taux de sénescence avec la température mais cette variabilité est considérablement réduite lorsqu'on tient compte de l'importance de l'enneigement au sol entre 2 dates d'observation. Dans les cas où l'enneigement est important (supérieur à la moitié de la durée de la période), la vitesse de sénescence est doublée environ. Apparemment, on ne note pas de différences entre parcelles et années.

En l'absence d'enneigement important, la plupart des feuilles restent vertes jusqu'à atteindre la somme de 250 degrés-jours, ce qui correspond à des durées très variables selon les températures moyennes journalières (de 1 mois à 3 mois).

TABLEAU 3

Coefficient des régressions pour l'élongation des feuilles $: e=\alpha e^{\beta \theta}$ et pour le rythme d'apparition des feuilles : $e=\alpha(\theta+\beta)$ à l'automne (a) et $e=\alpha(\log \theta+\beta)$ au printemps $(p)$.

Regression coefficients of lamina extension $: e=\alpha e^{\beta \theta}$ and leaf appearance rate $: e=\alpha(\theta+\beta)$ in autumn (a) and $e=\alpha(\log \theta+p)$ in spring (p) .

\begin{tabular}{|c|c|c|c|c|c|c|c|c|}
\hline \multirow{2}{*}{$\begin{array}{c}\text { Parcelles }(B, M) \\
\text { saisons (a, p) } \\
\text { dose d'azote } \\
(0 N, 60 N)\end{array}$} & \multirow[t]{2}{*}{$\begin{array}{l}\text { Nombre } \\
\text { d'années }\end{array}$} & \multirow[t]{2}{*}{$\begin{array}{c}\text { Nombre } \\
\text { de } \\
\text { données }\end{array}$} & \multicolumn{3}{|c|}{ Croissance des feuilles } & \multicolumn{2}{|c|}{$\begin{array}{c}\text { Rythme d'apparition } \\
\text { des } \\
\text { feuilles }\end{array}$} & \multirow[b]{2}{*}{$\mathrm{r}$} \\
\hline & & & $\alpha$ & $\beta$ & $\mathrm{r}$ & $\alpha$ & $\beta$ & \\
\hline $\mathrm{B}$ (a) $0 \mathrm{~N}$ & 3 & 7 & 0,193 & 0,226 & $0,784(0,05)$ & 0,69 & $-1,16$ & $0,931(0,001)$ \\
\hline$B(p) 0 N$ & 3 & 9 & 0,194 & 0,387 & $0,987(0,001)$ & 4,20 & $-0,20$ & $0,874(0,001)$ \\
\hline B (a) $60 \mathrm{~N}$ & 2 & 5 & 0,217 & 0,295 & $0,836(0,05)$ & 0,94 & $-1,12$ & $0,990(0,001)$ \\
\hline B (p) $60 \mathrm{~N}$ & 3 & 12 & 0,215 & 0,406 & $0,987(0,001)$ & 5,14 & $-0,24$ & $0,853(0,001)$ \\
\hline$M(a) \quad 0 N$ & 2 & 6 & 0,265 & 0,129 & $0,941(0,01)$ & 0,64 & $-2,15$ & $0,989(0,01)$ \\
\hline$M(p) 0 N$ & 2 & 6 & 0,301 & 0,233 & $0.885(0,01)$ & 4,26 & $-0,36$ & $0,965(0,001)$ \\
\hline$M(a) 60 N$ & 1 & 3 & & & & & & \\
\hline$M(p) 60 N$ & 2 & 5 & 0,284 & 0,267 & $0,906(0,05)$ & 4,69 & $-0,35$ & $0,795(0,05)$ \\
\hline
\end{tabular}



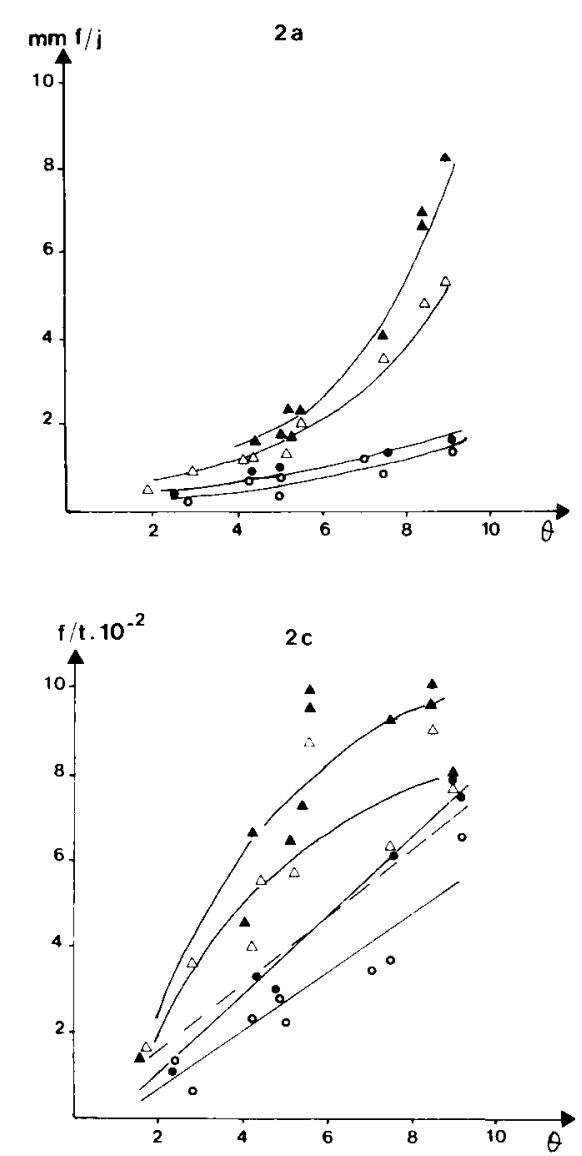

Figure 2

Elongation des limbes (fig. $2 a, b$ ) et rythme d'apparition des feuilles (fig. $2 c, d)$ en fonction de la température du sol $(-10 \mathrm{~cm})$ selon la fertilité de la parcelle : fig. $a$, c parcelle $B$, fig. $b, d$ parcelle $M$.

$O B$ (a) $0 N$ : parcelle $B$, points d'automne sans azote

$\triangle B$ (p) $O N$ : parcelle $B$, points de printemps sans azote

- $B$ (a) $60 \mathrm{~N}$ : parcelle $B$, points d'automne avec azote

$\triangle B$ (p) $60 \mathrm{~N}$ : parcelle $B$, points de printemps avec azote

$O M(a) O N)$

$\triangle M(p) \quad 0 N)$ même signification que pour $B$

$O M(a) 60 N($

$\Delta M(p) 60 \mathrm{~N}$

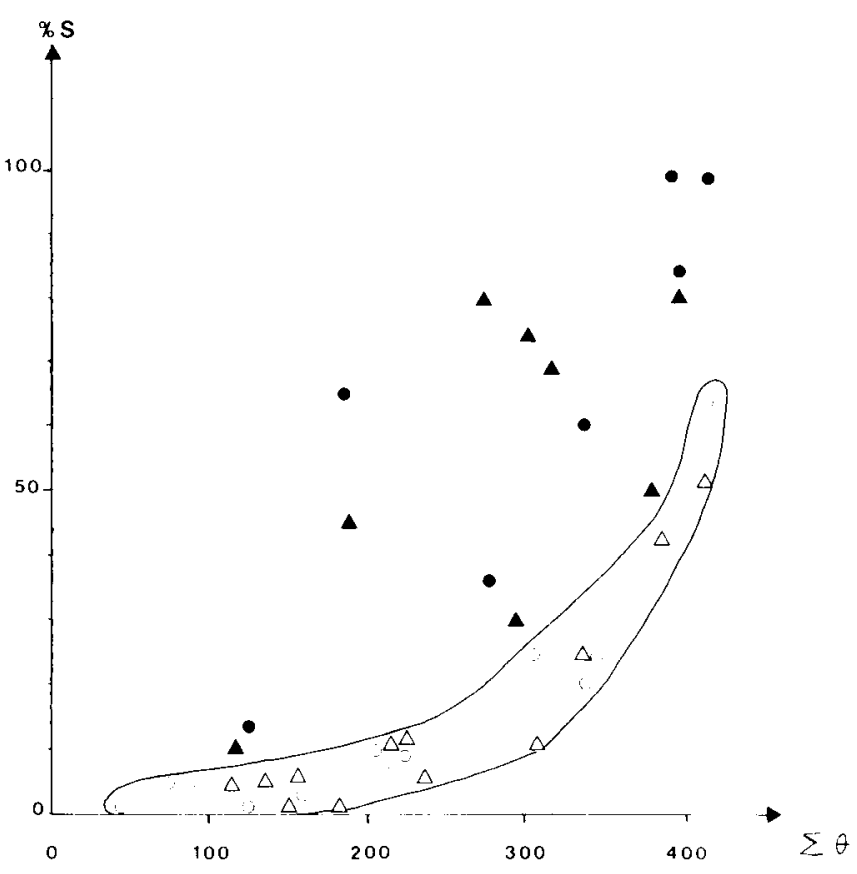

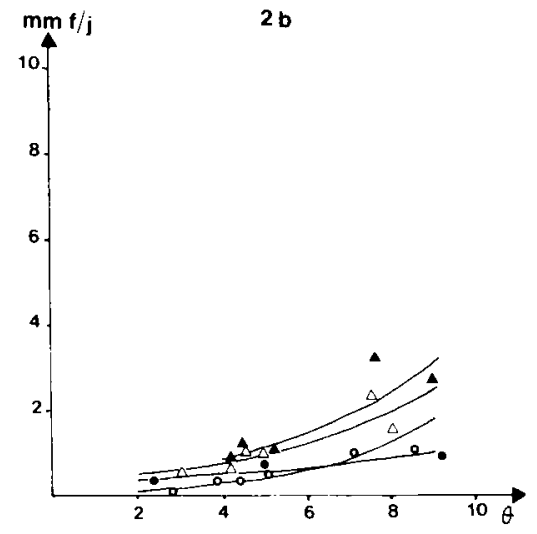

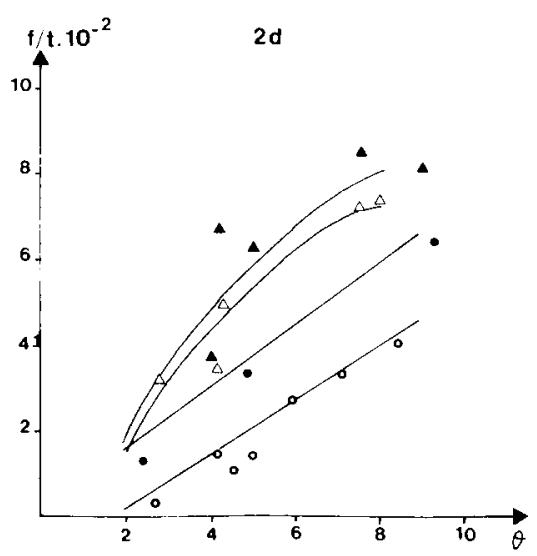

Lamina extension (fig. $2 a, b$ ) and rate of leaf appearance (fig. $2 c, d$ ) in relation to temperature according to soil fertility level : fig. $a, c$ plot $B$, fig. $b, d$ plot $M$.

$O B$ (a) $0 N$ : plot $B$, autumn data without nitrogen

$\triangle B$ (p) $O N$ : plot $B$, spring data without nitrogen

- $B$ (a) $60 \mathrm{~N}$ : plot $B$, autumn data with nitrogen

$\triangle B$ (p) $60 \mathrm{~N}$ : plot $B$, spring data with nitrogen

$O M(a) \quad 0 N$

$\triangle M(p) \quad 0 N$ ) same code as $B$

- $M(a) 60 N($

$\triangle M(p) 60 N$
Figure 3

Taux de sénescence (S) en fonction de la température aérienne (la température est cumulée à partir de la date de baguage de la feuille $T_{0}$ jusqu'aux dates d'observations suivantes $T_{1}$ et éventuellement $T_{2}$ et $T_{3}$ ).

$S=$ longueur cumulée de feuilles jaunes et sèches.

$$
\text { longueur totale de feuilles }
$$

Observations pour lesquelles l'enneigement a été inférieur à environ la moitié du temps écoulé entre $t_{n}$ et $t_{n}+1(\triangle \bigcirc)$ et supérieur

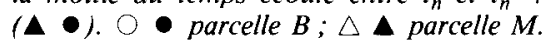

Senescence rate (S) in relation to air temperature (the temperature is cumulated from the date of leaf identification to the next observations $T_{l}, T_{2}$, or $T_{3}$ ).

$S=$ cumulated length of yellow and dead leaves.

$$
\text { total length of leaves }
$$

Observations of snow cover inferior $(\triangle O)$ or superior $(\Delta \bullet)$ at half duration between $t_{n}$ and $t_{n}+1, \bigcirc \bullet$ plot $B, \triangle \Delta$ plot $M$. 


\section{Tallage}

Dans les prairies permanentes où nous avons travaillé, les touffes de dactyle sont de dimensions et de densité fort variables de telle sorte qu'il est difficile d'estimer un nombre de talles par $\mathrm{m}^{2}$. Néanmoins, nous avons contrôlé à une date donnée le nombre de talles pour des touffes de dimensions variables. La figure 4 montre que plus la dimension de la touffe est importante moins le nombre de talles (ramené à l'unité de surface) est élevé. Ceci s'explique en partie par le fait que nous avons mesuré la circonférence des touffes à leur base et que plus la touffe est petite, plus la surface réelle, occupée par les talles comptabilisées, est supérieure à celle mesurée. Nous pouvons comparer les densités observées à celles de peuplements monospécifiques seulement pour des touffes de dimension importante où les "effets de bordure " sont réduits. Dans ces cas, nous observons encore des densités importantes $\left(10000\right.$ à 15000 talles par $\left.\mathrm{m}^{2}\right)$ ce qui est très supérieur aux données couramment rencontrées par ailleurs à la même période : 8000 sur ray-grass Anglais (DAVIES \& SIMONS, 1979), 2000 à 3000 sur ray-grass d'Italie (WILMAN \& OJUEDERIE, 1978), 3000 sur fétuque élevée (LEMAIRE, 1985).

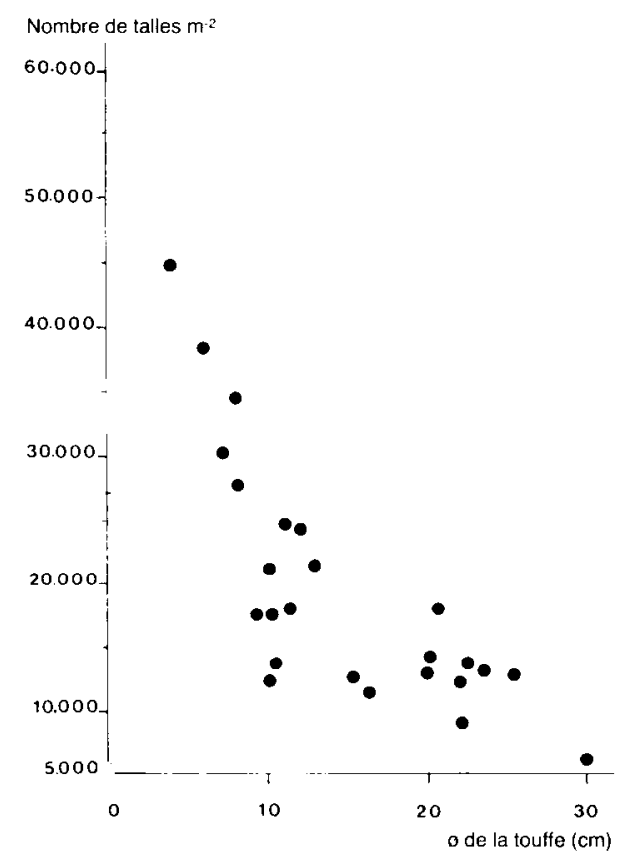

Figure 4

Relation entre densité de talles et circonférence d'une touffe de dactyle.

Relationship between tiller density and tuft circumference.

La figure 5a montre des variations importantes du nombre de talles selon les traitements (indice $100 \mathrm{au}$ premier contrôle de chaque année et traitement). Ce nombre est en général augmenté avec un apport d'azote. L'observation de la dynamique du nombre de talles montre une diminution en milieu d'hiver, une augmentation en mars-avril et une chute fin avril de telle sorte que le nombre de talles en fin d'hiver est égal ou supérieur à celui de départ. Pour les placettes défoliées, le nombre de talles est inférieur ce qui confirme les résultats de Davies (1974).

Nous avons calculé à chaque date les valeurs du «Site Filling » (Fs) défini par DaviEs (1974) comme le rapport entre la vitesse de tallage (talle par talle et par jour) et le rythme d'apparition des feuilles (feuille par talle et par jour). Nous observons que à une date donnée, les valeurs du Site Filling des différents traitements sont voisines (fig. 5b) alors que celles concernant le nombre de talles sont dispersées (fig. 5a). Cette observation montre que des facteurs autres que climatiques, régissant le nombre de talles, interviennent sur le rythme d'apparition des feuilles ce qui est particulièrement net pour l'azote.

En novembre, les valeurs de Fs légèrement positives ou nulles indiqueraient que les bourgeons de talles ne se développeraient pas normalement. Cette observation est à mettre en relation avec la forte densité de talles qui réduirait la vitesse de tallage (DAVIES \& THOMAS, 1983 ; JACQUARD, 1984). En décembre et janvier, les valeurs négatives de Fs correspondent à une baisse du nombre de talles qu'on peut interpréter par leur destruction par le froid. Début mars, les valeurs de Fs redeviennent positives et on atteint des valeurs supérieures ou égales à celles observées à l'automne. Début avril, on observe le maximum de talles mais la valeur théorique maximale du Site Filling $(0,481)$ est rarement atteinte. Après cette date, nous interprétons la mortalité importante de talles par la concurrence (le nombre de talles étant à son maximum) et par l'autoombrage important (THOMAS \& NORRIS, 1981), les feuilles les plus longues mesurant $100 \mathrm{~mm}$ en moyenne.

Le fait que le nombre de talles augmente systématiquement au mois de mars conforte l'hypothèse que nous avions émise précédemment à propos de l'élongation des feuilles. Compte tenu de leur date d'apparition, ces talles n'ont sans doute pas été exposées à des températures basses suffisamment longtemps (BRERETON et al., 1985) de telle sorte qu'elles restent à l'état végétatif.

\section{E. Croissance par touffe}

A chaque date d'observation, nous avons mesuré la longueur cumulée des feuilles apparues depuis l'observation précédente pour les 20 touffes de chacun des traitements. Cette mesure sous-estime la croissance car on ne tient pas compte des feuilles présentes à la date d'observation précédente et dont la croissance s'est poursuivie. Cette sous-estimation est plus importante en milieu d'hiver qu'en début ou fin dans la mesure où le rythme d'apparition des feuilles est lent, et les feuilles restent vertes sur une longue période.

Cette réserve étant faite, l'intérêt de cette mesure est d'intégrer les effets des 3 composantes que nous avons présentées ci-dessus (rythme d'apparition et élongation des feuilles, tallage). Sur la figure 6 , on retrouve très bien pour chaque parcelle les effets du stade physiologique et de l'azote ainsi que de la défoliation pour la parcelle B. La comparaison des pentes des droites de régression des parcelles $B$ et $M$ (légende de la fig. 6) montre des différences d'environ $30 \%$ en début d'hiver et de $40 \%$ en fin d'hiver. Nous pouvons noter que fin avril l'augmentation de l'élongation observée 

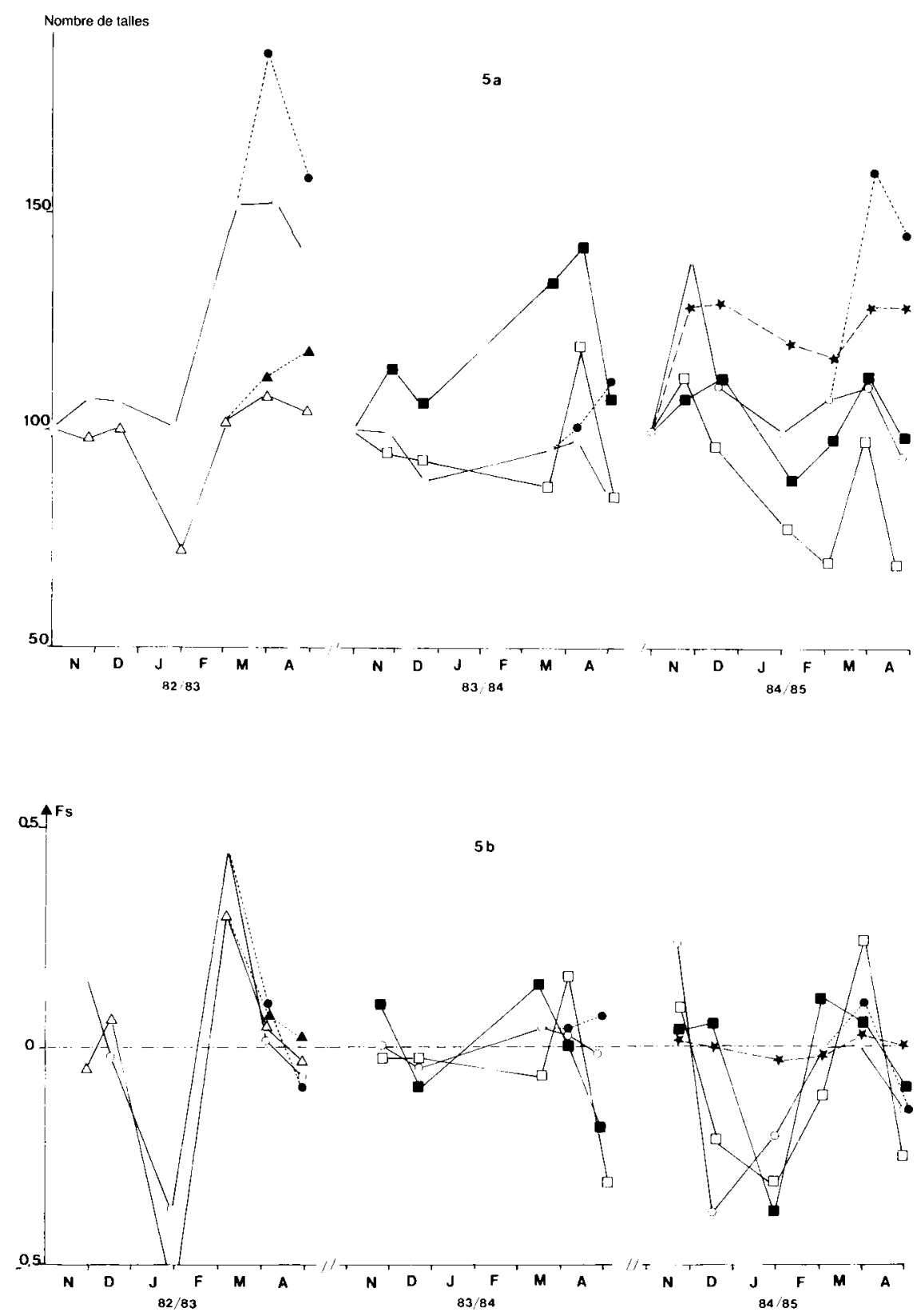

Figure 5

Evolution du tallage

$5 a:$ nombre de talles (indice 100 au premier contrôle)

$5 b:$ vitesse de tallage/rythme d'apparition des feuilles

$O B(a+p) 0 N ; \bullet B(p) 60 N ; \square B(a+p) 60 N ; \square B$ $(a+p) 60 N P ; \triangle M(a+p) 0 N ; \Delta M(p) 60 N ; \star M(a+p)$ $60 \mathrm{~N}$.

se produit malgré la baisse du nombre de talles. Ce phénomène est alors compensé par l'élongation exponentielle des limbes en fonction de la température.

\section{DISCUSSION}

Nous discuterons successivement les effets du climat (température, neige), de la nutrition azotée et phosphopotassique et de la défoliation. Puis nous proposerons un schéma récapitulatif des effets de l'ensemble de ces facteurs sur les différentes composantes de la croissance du dactyle (fig. 7).
Tillering evolution

$5 a$ : tiller number (index 100 for the first observation) $5 b$ : site filling.

$\bigcirc B(a+p) 0 N: \bullet B(p) 60 N ; \square B(a+p) 60 N ; \square B$ $(a+p) 60 N P ; \Delta M(a+p) 0 N ; \Delta M(p) 60 N ; \star M(a+p)$ $60 \mathrm{~N}$.

\section{A. Climat}

Les observations présentées montrent que la croissance et le développement des graminées sont simplement ralentis pendant l'hiver. L'effet du stade physiologique apparaît déterminant sur la réponse à la température pour la vitesse d'élongation des feuilles et leur rythme d'apparition. La période de changement que nous avons située au début février est voisine de celles déterminées plus précisément dans d'autres conditions : mi-janvier à fin janvier sur ray-grass Anglais en Grande-Bretagne (THOMAS \& NORRIS, 1977, 1979 ; PARSON \& ROBSON, 1980 ; VINE, 1983) ; tout début 

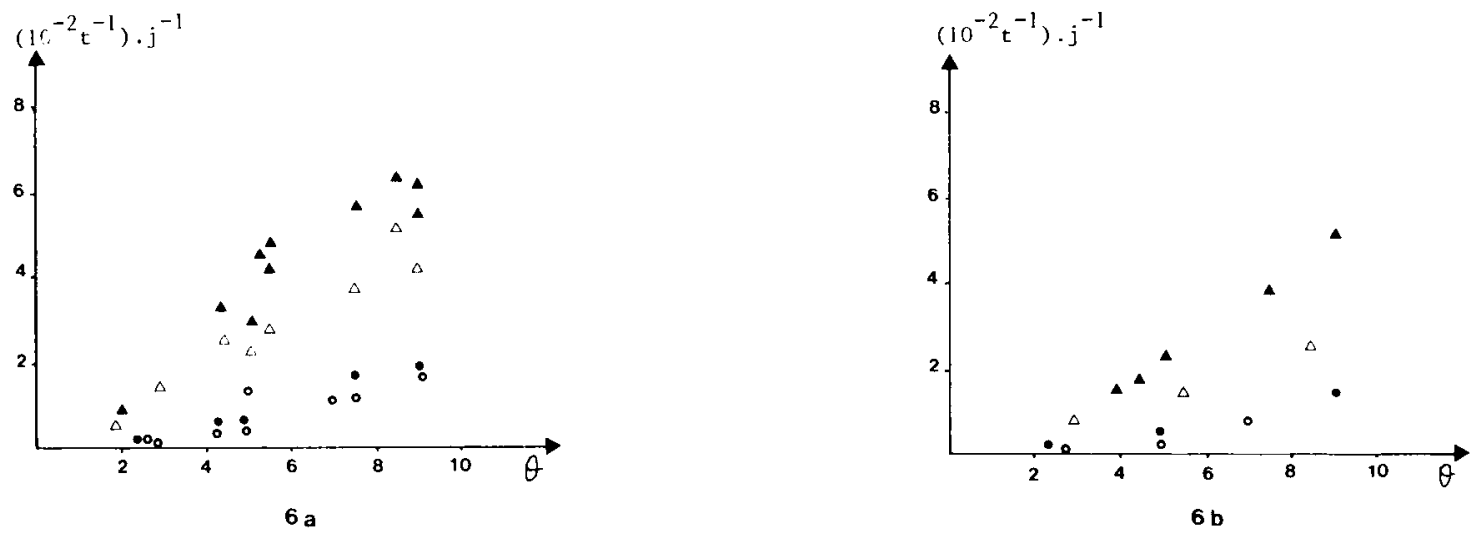

Figure 6

Elongation par talle et par jour (pour 100 talles présentes à la lre observation) en fonction de la température du sol selon la fertilité de la parcelle : fig. $6 a$ parcelle $B$; fig. $6 b$ parcelle $M$ (pour légende $c f$. fig. 2 ).

Les pentes et coefficients de corrélation des droites de régression sont respectivement :

0.20 et $0.85(0.01)$ pour $B$ (a) $0 N: 0.59$ et $0.92(0.001)$ pour $B$ (p) $0 N$

0.27 et $0.96(0.01)$ pour $B$ (a) $60 \mathrm{~N}: 0.76$ et $0.88(0.001)$ pour $B(p) 60 \mathrm{~N}$

0.26 et $0.96(0.01)$ pour $B(a) 60 \mathrm{NP}: 0.46$ et $0.87(0.01)$ pour $B(p) 60 \mathrm{NP}$

0.19 et $0.94(0.1)$ pour $M($ a) $0 N: 0.25$ et $0.96(0.05)$ pour $M(p) 60 \mathrm{NP}$

0.17 et $0.99(0.01)$ pour $M(a) 60 \mathrm{~N}: 0.46$ et $0.87(0.01)$ pour $M(p) 60 \mathrm{~N}$

Lamina extension per tuft per day (for 100 tillers at the first observation) in relation to soil temperature according to plot soil fertility level : fig. $6 a$ plot $B ;$ fig. $6 b$ plot $M$ (for references $c f$. fig. 2$)$.

Slopes and correlation coefficients of regression lines respectively:

0.20 and $0.85(0.01)$ for $B$ (a) $0 N \quad: 0.59$ and $0.92(0.001)$ for $B$ (p) $0 N$ 0.27 and $0.96(0.01)$ for $B$ (a) $60 \mathrm{~N}: 0.76$ and $0.88(0.001)$ for $B$ (p) $60 \mathrm{~N}$

0.26 and $0.96(0.01)$ for $B$ (a) $60 \mathrm{NP}: 0.46$ and $0.87(0.01)$ for $B(p) 6 N P$

0.19 and $0.94(0.1)$ for $M(a) \quad 0 N: 0.25$ and $0.96(0.05)$ for $M(p) \quad 0 N$

0.17 and $0.99(0.01)$ for $M(a) 60 \mathrm{~N}: 0.46$ and $0.87(0.01)$ for $M(p) 60 \mathrm{~N}$

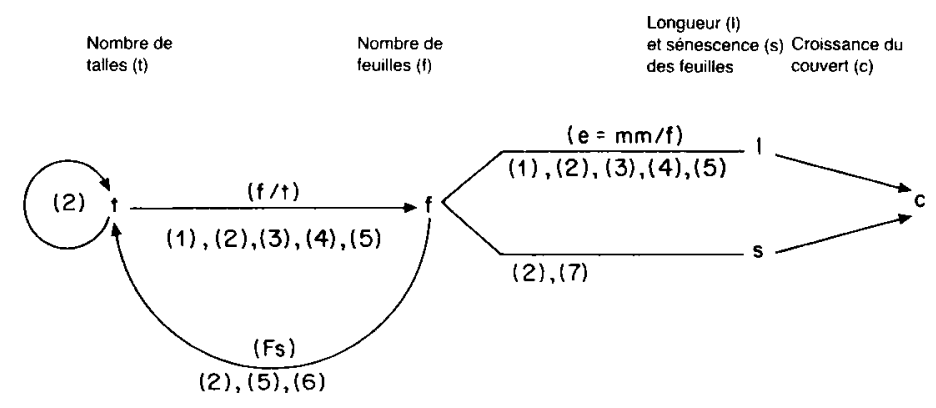

Figure 7

Schématisation des principaux effets des différents facteurs sur la morphogenèse du dactyle. (I) stade physiologique, (2) température, (3) nutrition azotée, (4) nutrition phosphopotassique, (5) défoliation, (6) compétition intraspécifique (entre talles), (7) neige. (Fs) : site filling.

février sur fétuque élevée au centre de la France (LEMAIRE, 1985).

Nous n'avons noté d'effet net de l'enneigement que sur la vitesse de sénescence des feuilles. Il se peut aussi que la couverture neigeuse limite la mortalité de talles dans le cas de températures aériennes fortement négatives. Nous n'avons repéré nettement ce phénomène que l'hiver 81/82 (DURU, 1983).

\section{B. Effet de l'azote}

D'une manière générale, l'effet de l'azote est d'autant plus important que les conditions de crois-
Schematic representation of different factors affecting cocksfoot morphogenesis: (1) physiological stage, (2) temperature, (3) nitrogen nutrition, (4) phosphopotassic nutrition, (5) defoliation, (6) intraspecific competition (between tillers), (7) snow. (Fs) : site filling. sance sont plus favorables : après mi-février, et parcelle de bonne fertilité. Il est à remarquer que les niveaux de nutrition azotée ont été sans doute voisins entre années puisque les différents paramètres de la croissance sont assez bien corrélés à la température même sans apport d'azote. L'azote augmente dans tous les cas la vitesse d'élongation des feuilles, mais son effet sur le rythme d'apparition des feuilles est discuté. Juste après les semis, cet effet est très important (THOMAS, 1983) mais dans des couverts végétaux installés (ray-grass anglais, fétuque élevée), on note des réductions de 10 à $30 \%$ du rythme d'apparition 
des feuilles pour les situations sans apport d'azote relativement à celles en recevant (RYLE, 1964 ; WILMAN et al., 1976 ; WILMAN \& MOHAMED, 1980 ; JOY PEARCE et WILMAN, 1984). Nos observations sont voisines de celles rapportées ici mais différentes de celles de LEMAIRE (1985) et d'autres auteurs dont WITHEHEAD (1970). Une des hypothèses plausibles est que les délais entre les apports d'azote et les observations sont parfois très différents selon les expérimentations.

La confrontation à d'autres situations des résultats obtenus sur la parcelle B avec fertilisation azotée montre des vitesses d'élongation des feuilles en fonction de la température très voisines en fin d'hiver et début de printemps et ce pour des espèces différentes : ray-grass anglais (THOMAS \& NORRIS, 1977, 1979) ; fétuque, fléole, ray-grass d'Italie (PEACOCK, 1976). Par contre, dans nos conditions, elles sont toujours très inférieures dans la première partie de l'hiver. Le rythme d'apparition des feuilles est toujours égal ou supérieur à celui observé dans les situations précitées. La densité de talles observées à l'automne étant très supérieure aux situations couramment rencontrées, le nombre de feuilles par $\mathrm{m}^{2}$ est donc lui aussi très supérieur (la sénescence étant voisine). Ces observations peuvent s'interpréter par une compétition pour l'utilisation des assimilats en faveur du maintien d'un nombre de talles important plutôt que d'une croissance des feuilles.

\section{Effet de la fertilité du sol}

Peu de résultats sont disponibles concernant l'effet de la nutrition phosphopotassique sur les différentes composantes de la croissance. Nous avons globalement observé cet effet sur l'élongation par talle et ses composantes (principalement élongation des limbes et rythme d'apparition des feuilles). De même que pour l'azote, l'effet de la fertilité du sol est particulièrement important lorsque les conditions de croissance sont favorables (température, azote).

\section{Effet de la défoliation}

L'effet de la défoliation telle qu'elle a été pratiquée, est important sur l'élongation cumulée par talle (fig. 6). Nous avons observé des effets nets sur le rythme d'apparition des feuilles ce qui confirme les résultats de Davidson \& MilThorpe (1966), Davies (1974), et sur le tallage (DAVIES, 1974 ; WILMAN et al., 1976). Nous n'avons pas observé l'élongation des feuilles dans ces conditions mais DAviEs \& SimONS (1979) ont montré qu'un retard de la dernière coupe d'automne diminuait la longueur des feuilles produites ultérieurement. Il semble que dans ces conditions le taux de glucides solubles à la base des feuilles influe sur la croissance des feuilles après défoliation (DAVIDSON \& MilThORPE, 1966).

Le comportement du dactyle dans les prairies, telles qu'elles sont pâturées, doit vraisemblablement être intermédiaire entre nos observations sans défoliation et avec défoliation puisque en simulation de pâturage continu KING et al. (1984) ont observé des vitesses de croissance quasiment constantes pour un indice foliaire compris entre 2 et 4,5 ce qui correspond approximativement à une hauteur d'herbe de 3 à $7 \mathrm{~cm}$ (GRANT et al., 1983) alors que nos prélèvements ne laissaient que $1 \mathrm{~cm}$ environ à la base des feuilles.

\section{CONCLUSION}

L'étude de l'écophysiologie du dactyle montre que les composantes de sa croissance dépendent au moins en grande partie de la nutrition phosphopotassique compte tenu des différences observées en condition de nutrition azotée non limitante (rappelons que dans les conditions de notre étude, les différences de nutrition phosphopotassique entre les parcelles proviennent de différences de fertilité chimique du sol). Pour notre observation qui se rapproche le plus d'une mesure de la croissance (longueur cumulée des feuilles nouvellement apparues entre 2 observations), nous observons des réductions de $30 \%$ (début hiver) et $40 \%$ (fin hiver) pour la parcelle où la fertilité phosphopotassique est moindre. On peut donc penser que les différences de croissance observées globalement au niveau du couvert végétal dépendent de la nutrition phosphopotassique. On ne peut toutefois pas exclure l'hypothèse d'un effet de la composition botanique. En effet, des différences entre espèces ou cultivars ont été mises en évidence en ce qui concerne la réponse du couvert végétal ou des feuilles à la température (PEACOCK, 1976 ; LeMAIRE \& SAlETTE, 1984b). Toutefois, les différences enregistrées sont moindres que celles que nous avons signalées ci-dessus, ce qui confirmerait l'effet prépondérant de la nutrition phosphopotassique dans les situations que nous avons étudiées.

Il est remarquable de constater que, pour un niveau de nutrition minérale donné, la croissance globale des touffes de dactyle est bien dépendante de la température (la relation est assimilable à une droite) alors que nous avons montré que ses effets étaient complexes et différenciés sur la croissance des feuilles, leur rythme d'apparition et le tallage (en particulier, le nombre de talles vivantes peut doubler en l'espace de 2 mois). Cette remarque vaut pour les très faibles températures, les touffes soumises à défoliation et les situations carencées en acide phosphorique et potasse. On peut penser que les régulations qui s'établissent au sein de la touffe notamment entre nombre de talles et longueur de leurs feuilles (GILLET, 1980), ou bien élongation des feuilles et type de talles (BRERETON et al., 1985) tamponnent les effets complexes de la température sur la croissance et le nombre d'organes.

Reçu le 14 mai 1985. Accepté le ler octobre 1986. 


\section{RÉFÉRENCES BIBLIOGRAPHIQUES}

Balent G., Duru M., 1984. Influence des modes d'exploitation sur les caractéristiques et l'évolution des surfaces pastorales : cas des Pyrénées Centrales. Agronomie, 4 (2), 113-124.

Behague J. J., 1978. The seasonal discrepancies between potential and actual grass growth. An essay of explanation by ecophysiological constraints. Proc. 7th General Meeting European Grassland Federation Ghent. Session 2, 1-19.

Brereton A. J., Carton O. T., O'Keeffe W. F., 1985. Tissue turnover in perennial ryegrass (Lolium perenne L.) during winter. Ir. J. Agric. Res, 24, 49-62.

Daget Ph., Poissonnet J., 1971. Une méthode d'analyse phytologique des prairies. Ann. Agron., 22 (1), 5-41.

Davidson J. L., Milthorpe F. L., 1966. Leaf growth in Dactylis glo merata following defoliation. Ann. Bot., 30, 118, 173-184.

Davies A., 1974. Leaf tissue remaining after cutting and regrowth in perennial ryegrass. J. agric. Sci., Camb., 82, 165-172.

Davies A., Simons R. G., 1979. Effect of autumn cutting regime on development morphology and spring growth of perennial ryegrass. J. Agric. Sci., Camb., 92, 457-469.

Davies A., Thomas H., 1983. Rates of leaf and tiller production in young spaced perennial ryegrass plants in relation to soil temperature and solar radiation. Ann. Bot., 57, 591-597.

Duru M., 1983. Caractérisation de la pousse hivernale des prairies permanentes dans les Pyrénées Centrales. Agronomie, 3 (5), 461-472.

Duru M., 1985. Winter and spring growth variability of grazed meadow. The case of the Pyrenees mountains. Proceedings of the $X V^{e}$ Grassland International Congress, Kyoto, 24-31/8 (à paraître), 595-597.

Duru M., Gibon A., Langlet A., Flamant J.-C., 1979. Recherche sur les problèmes pastoraux pyrénées in $X^{e}$ journée du Grenier de Theix, "Pâturage d'altitude et parcours méditerranéens », 232-256.

Duru M., Gibon A., 1981. Disponibilités hivernales pour le pâturage dans les Pyrénées Centrales. Premières observations sur les prés de fauche. C. R. Séances Acad. Agric. Fr., 2, 186-196.

Gibon A., 1981. Pratiques d'éleveurs et résultats d'élevage dans les Pyrénées Centrales. Thèse Docteur Ingénieur, I.N.A.-P.G., 106 pages.

Lemaire G., 1985. Cinétique de la croissance d'un peuplement de fétuque élevée (Festuca arundinacea Schreb.) pendant l'hiver et le printemps. Effet des facteurs climatiques. Thèse de doctorat d'état. Université de Caen. 96 pages.

Lemaire G., Salette J., 1984b. Relation entre dynamique de croissance et dynamique de prélèvement d'azote pour un peuplement de graminées fourragères. II. Etude de la variabilité entre génotypes. Agronomie, 4 (5), 431-436.
Parson A. J., Robson M. J., 1980. Seasonal changes in the physiology of S24 perennial ryegrass (Lolium perenne L.) 1. Response of leaf extension to temperature during the transition from vegetative to reproductive growth. Ann. Bot. 46, 435-444.

Peacock J. M., 1975. Temperature and leaf growth in Lolium perenne. III. Factors affecting seasonal differences. J. appl. ecol. 12, 685-697.

Peacock J. M., 1976. Temperature and leaf growth in four grass species. J. appl. ecol. 13, 225-232.

Robson M. J., 1974. The effect of temperature on the growth of S170 tall fescue. III. Leaf growth and tiller production as affected by transfer between contrasting regimes. J. of Appl. Ecol., 11, 265279.

Ryle G. J. A., 1964. A comparaison of leaf and tiller growth in seven perennial grasses as influenced by nitrogen and temperature. J. of Br. Grass. Soc. 19, 281-290.

Thomas H., 1983. Analysis of the nitrogen response of leaf extension in Lolium temulentum seedlings. Ann. Bot. 51, 363-371.

Thomas H., Norris I. B., 1977. The growth responses of Lolium perenne to the weather during winter and spring at various altitude in mid-Wales. J. appl. Ecol., 14, 949-964.

Thomas H., Norris I. B., 1979. Winter growth contrasting ryegrass varieties at two altitudes in mid-Wales. $J$. of appl. Ecol., 16, 553565

Thomas H., Norris I. B., 1981. The influence of light and temperature during winter on growth and death in simulated swards of Lolium perenne. Grass and Forage Sci., 36, 107-116.

Vine D. A., 1983. Sward structure changes within a perennial ryegrass sward : leaf appearance and death. Grass and Forage Sci., 38, 23-242.

Whitehead D. C., 1970. The role of nitrogen in grassland productivity. Commonwealth Bureau Pastures and Field Crops, bulletin 48, 202 pages.

Wilman D., 1980. Early spring and late autumn response to applied nitrogen in four grasses. 1. Yield, number of tillers and chemical composition. J. agric. Sci., Camb., 94, 425-442.

Wilman D., Mohamed A. A., 1980. Early spring and late autumn response to applied nitrogen in four grasses. 2. Leaf development. J. agric. Sci., Camb., 94, 443-453.

Wilman D., Ojuederie B. M., 1978. The response of Italian ryegrass to nitrogen fertilizer at different times of year, with particular reference to residual effect. J. agric. Sci., Camb., 90, 479-494.

Wilman D., Koocheki A., Lwoga A. B., Droushiotis D., Shim J. S., 1976. The effect of interval between harvest and nitrogen application on the numbers and weights of tillers and leaves in four ryegrass varieties. J. agric. Sci., Camb., 87, 45-57. 